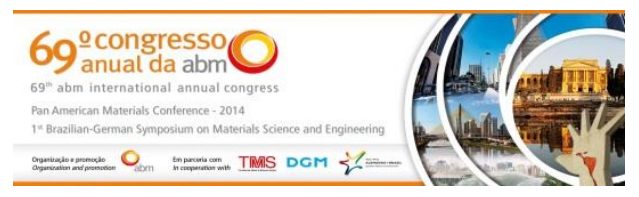

Tema: Fundição

\title{
CORRELAÇÃO ENTRE MICROESTRUTURA E DUREZA DA LIGA LIVRE DE CHUMBO Bi-1,5\%Ag*
}

\author{
Robson Aguiar de Macedo ${ }^{1}$ \\ Bismarck Luiz Silva² \\ José Eduardo Spinelli3
}

\begin{abstract}
Resumo
O estudo de ligas livres de $\mathrm{Pb}$ envolvendo o emprego de junções metálicas sob altas temperaturas tem recebido atenção especial, sendo desenvolvidas novas ligas com qualidade e desempenho adequados. Ligas $\mathrm{Bi}$-Ag consistem em alternativas promissoras para a substituição das ligas de solda contendo $\mathrm{Pb}$ para tais aplicações, por isso devem ser melhor caracterizadas sob o aspecto de estruturas brutas de fusão. Assim, o presente trabalho objetiva investigar a liga hipoeutética $\mathrm{Bi}-1,5 \% \mathrm{Ag}$ (\%em peso) solidificada unidirecionalmente sob regime transitório de fluxo de calor. Será analisada a influência dos parâmetros térmicos de solidificação como velocidade de solidificação $\left(V_{\mathrm{L}}\right)$, taxa de resfriamento $(\dot{T})$ gradiente de temperatura $(G)$ na microestrutura e na evolução de dureza da liga citada. Para determinação do perfil de dureza, foram realizados ensaios de dureza Vickers. Para obtenção e caracterização das microestruturas presentes no fundido $\mathrm{Bi}-1,5 \% \mathrm{Ag}$, fez-se o uso da microscopia ótica e da fluorescência de raios-x. Uma estrutura de dendritas facetadas de $\mathrm{Bi}$ circundadas pelo eutético $\mathrm{Bi}$-Ag prevaleceu ao longo do lingote $\mathrm{Bi}$ $1,5 \% \mathrm{Ag}$. Verificou-se um aumento gradativo dos espaçamentos dendríticos primário $\left(\lambda_{1}\right)$ e secundário $\left(\lambda_{2}\right)$ à medida que a distância da base refrigerada foi aumentada. Em geral, os valores de dureza diminuíram para maiores valores de $\lambda_{1}$ e $\lambda_{2}$.
\end{abstract}

Palavras-chave: Ligas Bi-Ag; Microestrutura; Solidificação; Dureza.

\section{CORRELATION BETWEEN MICROSTRUCTURE AND HARDNESS OF Bi-1.5wt\%Ag LEAD-FREE SOLDER ALLOY}

\begin{abstract}
The study of $\mathrm{Pb}$-free alloys involving the use of metallic connections under high temperatures has received special attention in the last few years, being developed new alloys with adequate quality and performance. Bi-Ag alloys appear as potential candidates to replace solder alloys containing $\mathrm{Pb}$. Hence, such alloys are needed to be better characterized considering their as-cast or assoldered microstructures. The aim of this study is to investigate a lead-free $\mathrm{Bi}-1.5 \mathrm{wt} \% \mathrm{Ag}$ solder alloy solidified unsteady-state upward unidirectional conditions. The influence of thermal parameters as tip growth rate $\left(V_{L}\right)$, cooling rate $(\dot{T})$ and thermal gradient $(G)$ on the final microstructure and hardness properties will be examined. A hardness Vickers tester was used in order to determine the hardness profile of the Bi-1.5wt\%Ag alloy. The directionally solidified microstructure was characterized by both light microscopy and fluorescence spectrometer. It was found that the as-cast structure was arranged by faceted Bi-rich dendrites sorrounded by a eutectic mixture $(\mathrm{Bi}+\mathrm{Ag})$. In general, the hardness values decrease for higher of $\lambda_{1}$ and $\lambda_{2}$ values.
\end{abstract}

Keywords: Bi-Ag alloys; Microstructure; Solidification; Hardness.

1 Graduando, Departamento de Engenharia de Materiais, UFSCar, São Carlos, SP, Brasil.

2 Doutorando, PPG-CEM, UFSCar, São Carlos, SP, Brasil.

3 Professor, Departamento de Engenharia de Materiais, UFSCar, São Carlos, SP, Brasil.

\footnotetext{
* Contribuição técnica ao 69ำ Congresso Anual da ABM - Internacional e ao 14 ENEMET - Encontro Nacional de Estudantes de Engenharia Metalúrgica, de Materiais e de Minas, 21 a 25 de julho de 2014, São Paulo, SP, Brasil.
} 


\section{INTRODUÇÃO}

A miniaturização é uma tendência inevitável das operações de conexões de componentes eletrônicos dedicados, por exemplo, a telefones celulares e outros aparelhos eletrônicos de uso pessoal. Esta tendência associada ao banimento contemporâneo de ligas à base de $\mathrm{Pb}$ [1-4] para operações de brasagem (soldering) são desafios atuais dos mais importantes para a indústria de montagem de microeletrônicos. Primeiramente deve ser ressaltada a ausência de estudos específicos envolvendo aspectos de processamento, microestrutura e propriedades para determinadas ligas alternativas ao emprego do chumbo em condições de alta temperatura, quais sejam: Au-Sn, Au-Ge, Zn-Al, Zn-Sn, Bi-Ag e Sn-Sb [5]. Segundo Watson e Castro [6], o aumento expressivo de aplicações envolvendo o emprego de sistemas microeletrônicos em condições extremas de temperatura e pressão, os quais certamente dependem fundamentalmente do uso de metais de adição livres de chumbo e conjuntos conectados confiáveis para suportar as condições citadas.

As ligas à base de bismuto são prováveis substitutas da liga $\mathrm{Pb}-5 \% \mathrm{Sn}$, uma vez que suas temperaturas típicas são consideradas aceitáveis (temperatura liquidus da liga hipoeutética $\mathrm{Bi}-1,5 \% \mathrm{Ag}: 265,5^{\circ} \mathrm{C}$ ), associadas às excelentes propriedades mecânicas desde que haja controle adequado da microestrutura. As ligas Bi-Ag possuem relativo baixo custo de fabricação, contudo baixos valores de condutividade térmica e elétrica. Estas ligas são, portanto, uma promessa entre as ligas alternativas de soldagem, embora estudos detalhados de solidificação em equilíbrio e fora de equilíbrio ainda sejam escassos.

Desta forma, a manipulação e controle dos parâmetros térmicos de solidificação ( $\mathrm{V}$, †ं e G) associados com a evolução microestrutural se tornam fundamentais no controle da qualidade da junta soldada [7,8]. Além disso, ensaios de dureza podem ser extremamente úteis para avaliar o desempenho mecânico das diferentes configurações microestruturais resultantes após a solidificação [9,10].

Portanto, o presente trabalho objetiva a realização do experimento de solidificação unidirecional ascendente da liga $\mathrm{Bi}-1,5 \% \mathrm{Ag}$ sob condições transitórias de extração de calor. Também devem ser determinados os parâmetros de solidificação ( $\mathrm{V} L$, Tं e G) e a evolução dos espaçamentos dendríticos $\left(\lambda_{1}, \lambda_{2}\right)$. Além disso, os perfis de dureza serão correlacionados com a microestrutura dendrítica, levando em consideração os efeitos do refino dendrítico da liga em estudo.

\section{PROCEDIMENTO EXPERIMENTAL}

O lingote da liga hipoeutética $\mathrm{Bi}-1,5 \% \mathrm{Ag}$ (composição nominal) foi obtido através da utilização do dispositivo de solidificação unidirecional vertical ascendente, cujo detalhamento do aparato experimental pode ser encontrado na literatura [11]. $O$ diagrama de fases do sistema $\mathrm{Bi}-\mathrm{Ag}$ pode ser considerado como do tipo eutético simples (veja Figura 1 a seguir), com longo patamar de formação de estrutura eutética e ponto de reação eutética para o teor de $97,4 \% \mathrm{Bi}$ e temperatura $262,5^{\circ} \mathrm{C}$. O dispositivo é composto por um sistema de aquecimento (resistências elétricas envolvidas por uma casca cilíndrica refratária) e por um conjunto lingoteira/chapa molde (aço carbono 1020) que são responsáveis pela fusão e solidificação do metal líquido, respectivamente.

\footnotetext{
* Contribuição técnica ao $69^{\circ}$ Congresso Anual da ABM - Internacional e ao 14ํㅡㄹ ENEMET - Encontro Nacional de Estudantes de Engenharia Metalúrgica, de Materiais e de Minas, 21 a 25 de julho de 2014, São Paulo, SP, Brasil.
} 

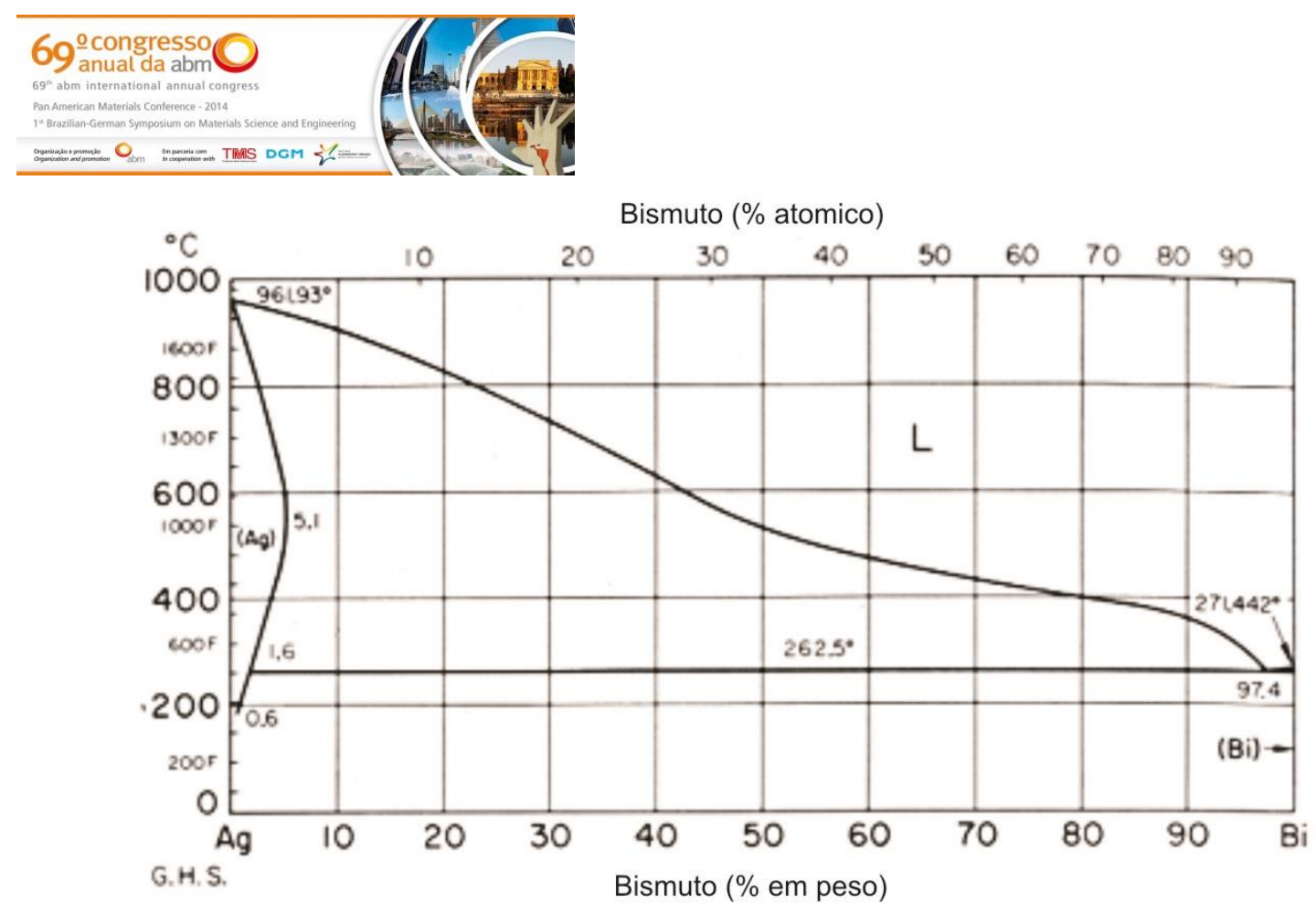

Figura 1. Diagrama de fases do sistema Bi-Ag.

O lingote fundido foi seccionado na direção longitudinal e depois transversalmente para obtenção das amostras de diversas posições ao longo do mesmo. As posições foram $5,15,30$ e $50 \mathrm{~mm}$ a partir da base refrigerada. As amostras foram lixadas sequencialmente por lixas de granulometria 150, 240, 320, 400, 500, 600, 1200 e 1500 mesh em lixadeiras manuais com refrigeração a água. Após lixadas e limpas, elas foram polidas com abrasivo, uma solução de alumina de $1 \mu \mathrm{m}$. Esta etapa foi realizada em politrizes rotativas, tornando assim a superfície a ser analisada pronta para o ataque químico. Por fim, as amostras foram atacadas quimicamente para revelação das microestruturas presentes no fundido. O reagente químico usado foi uma solução de $4 \%$ de $\mathrm{HNO}_{3}$ (ácido nítrico) e $96 \%$ de $\mathrm{C}_{2} \mathrm{H}_{5} \mathrm{OH}$ (álcool etílico). Um conjunto de microestruturas (transversais e longitudinais) foi registrado por meio da utilização do microscópio ótico Olympus BX41M-LED e do sistema de processamento de imagens Infinity Capture acoplado ao microscópio.

As medidas de espaçamentos dendríticos primários $\left(\lambda_{1}\right)$ foram realizadas segundo 0 método do triângulo [12,13], uma vez que as condições de solidificação com fluxo de calor transitório podem proporcionar um crescimento bastante irregular das dendritas. Para quantificar os espaçamentos dendríticos secundários $\left(\lambda_{2}\right)$ foi utilizado o método do intercepto [14]. As medições de $\lambda_{1}$ e $\lambda_{2}$ foram realizadas sobre a secção transversal (perpendicular à direção de extração de calor) e longitudinal do lingote $\mathrm{Bi}-1,5 \% \mathrm{Ag}$, respectivamente. Foram realizadas, em média, 25 a 40 medidas de $\lambda_{1}$ e $\lambda_{2}$ para cada posição analisada.

O durômetro utilizado nos ensaios de dureza Vickers (HV) é da marca Future-Tech modelo FM-800. As medidas de dureza (penetrador do tipo Vickers de diamante piramidal de base quadrada e carga de $1000 \mathrm{~g}$ ) foram feitas de acordo com a norma ASTM E384-11. Durante os ensaios, tomou-se o cuidado de cumprir o espaçamento mínimo necessário entre as identações e entre cada identação e a borda $(2,5 \mathrm{~d}$, onde "d" é a diagonal média da identação Vickers) [9]. Para cada posição selecionada ao longo do lingote fundido foram realizadas cerca de 10 medidas de dureza, totalizando 110 valores de HV.

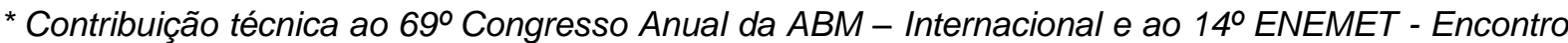
Nacional de Estudantes de Engenharia Metalúrgica, de Materiais e de Minas, 21 a 25 de julho de 2014, São Paulo, SP, Brasil.
} 


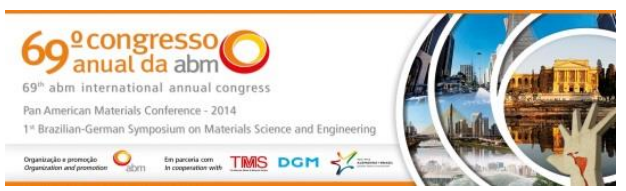

\section{RESULTADOS E DISCUSSÕES}

A Figura 2 mostra as curvas de resfriamento experimentais obtidas para a liga hipoeutética $\mathrm{Bi}-1,5 \% \mathrm{Ag}$, considerando o conjunto de termopares presente no fundido. É possível observar o histórico térmico monitorado durante o experimento para a liga em análise. Quando atingida a temperatura liquidus $\left(265,5^{\circ} \mathrm{C}\right)$, tem-se início a liberação de calor latente de fusão e após liberado, e o fim da solidificação conforme indicado na Figura 2.

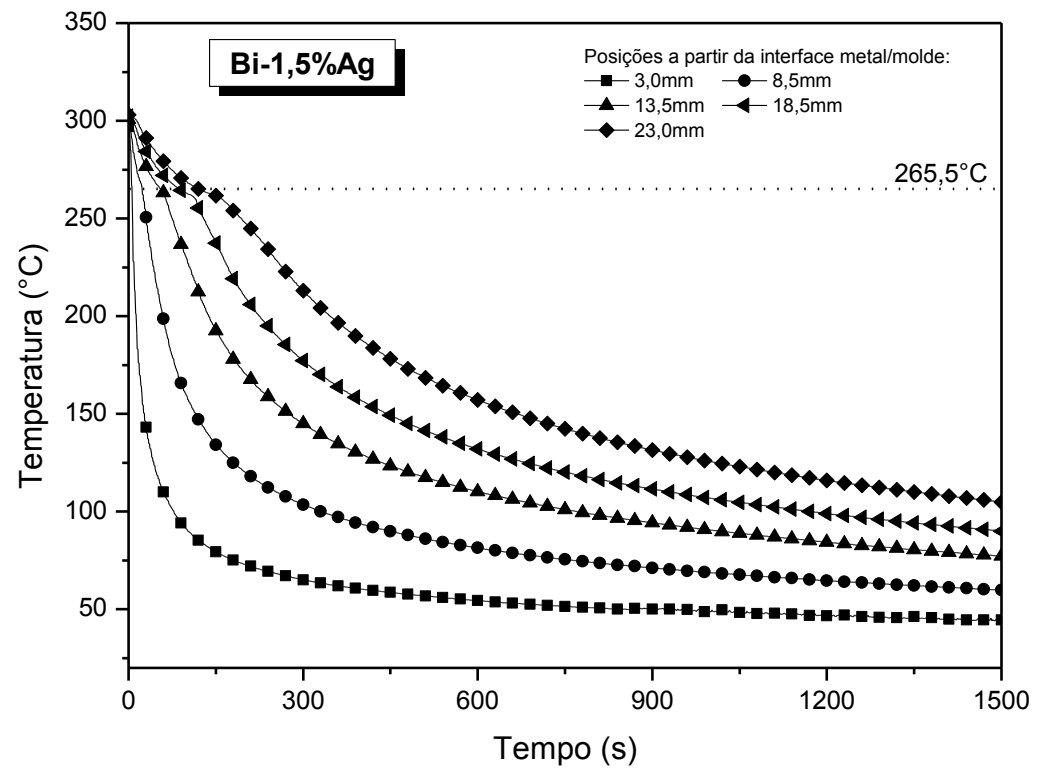

Figura 2. Curvas de resfriamento experimentais da liga hipoeutética Bi-1,5\%Ag solidificada unidirecionalmente.

A partir dos perfis térmicos foi possível mapear a passagem da isoterma liquidus em cada um dos termopares, permitindo relacionar a posição desta isoterma com tempo. A representação gráfica desse mapeamento para o sistema $\mathrm{Bi}-1,5 \% \mathrm{Ag}$ é mostrada na Figura 3a. Por meio da aplicação de técnicas matemáticas de ajustes de curvas aos pontos experimentais é possível representar a dependência funcional da isoterma liquidus com o tempo, utilizando-se uma função potência. A derivada dessa função em relação ao tempo resulta na velocidade de avanço dessa isoterma. As Figuras 3b, 3c e 3d mostram as evoluções da velocidade da isoterma liquidus $\left(V_{L}\right)$, da taxa de resfriamento $(\dot{T})$ e do gradiente térmico $(G)$ com a posição, respectivamente, ao longo do lingote $\mathrm{Bi}-1,5 \% \mathrm{Ag}$.

Os valores de $V_{L}$, $\dot{T}$ e $G$ diminuem para posições mais afastadas da interface metal/molde. Isso ocorre basicamente devido as novas resistências térmicas incorporadas ao longo do processo que são representadas pelas camadas sólidas que se formam continuamente durante a evolução da solidificação a partir da base do lingote.

* Contribuição técnica ao $69^{\circ}$ Congresso Anual da ABM - Internacional e ao 14ํㅡㄹ ENEMET - Encontro Nacional de Estudantes de Engenharia Metalúrgica, de Materiais e de Minas, 21 a 25 de julho de 2014, São Paulo, SP, Brasil. 


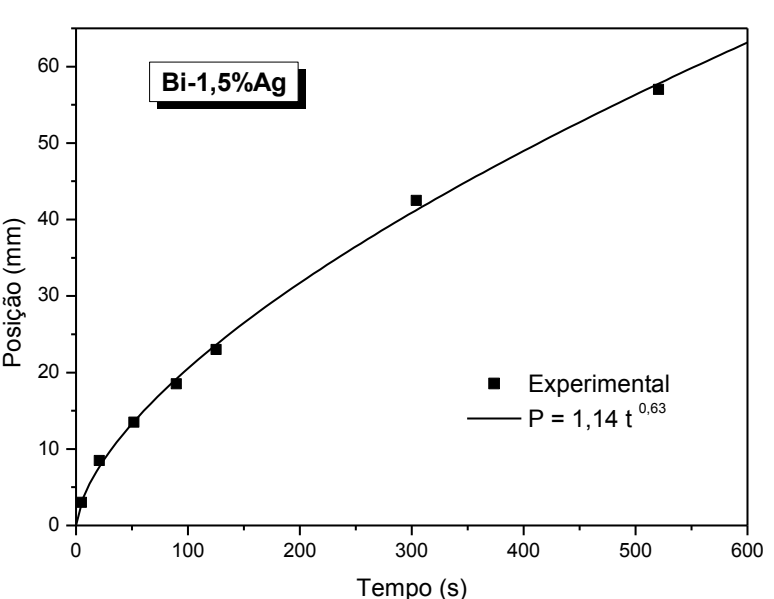

(a)

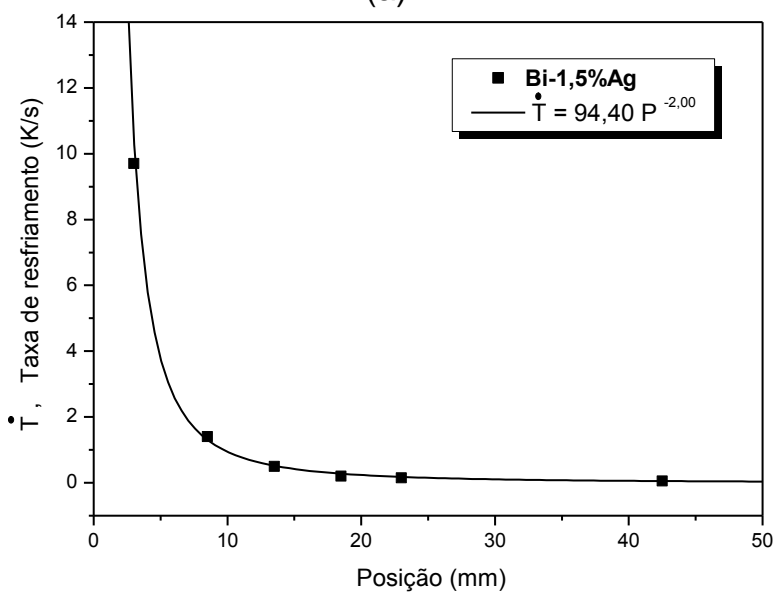

(c)

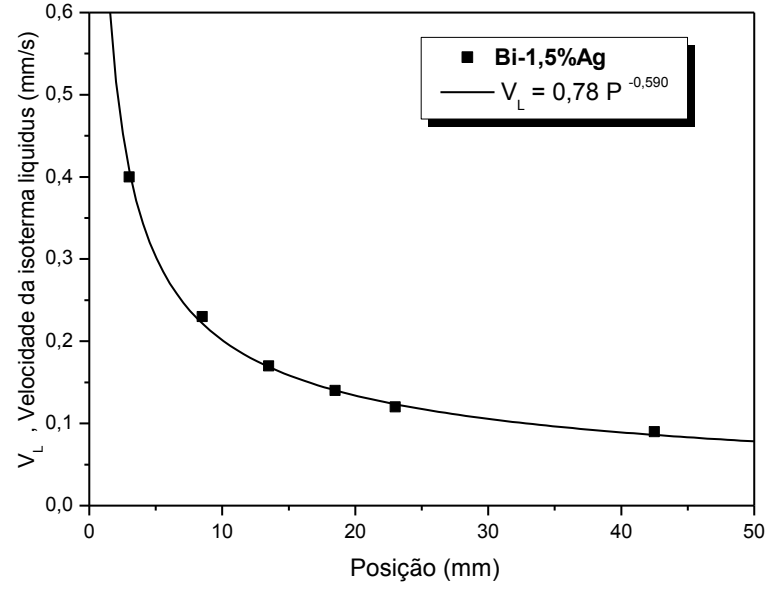

(b)

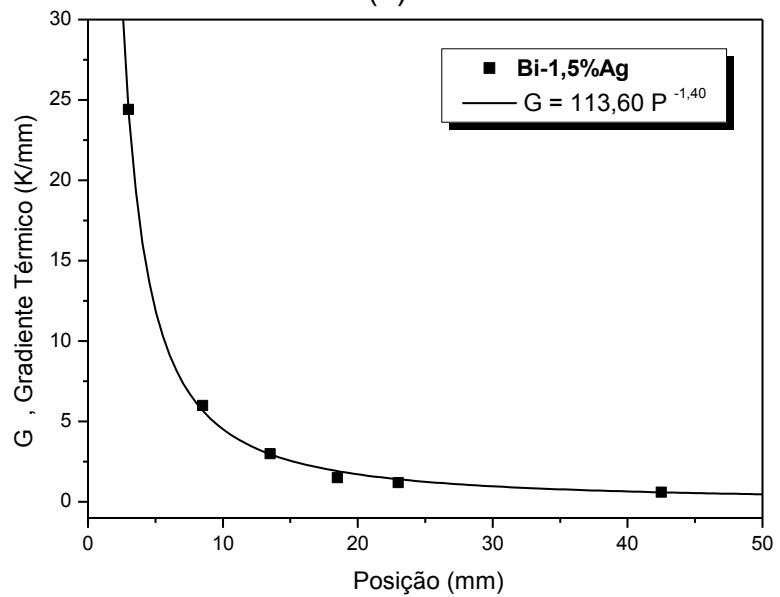

(d)

Figura 3. (a) Posição da isoterma liquidus a partir da interface metal/molde; (b) evolução do avanço da isoterma liquidus $\left(\mathrm{V}_{\mathrm{L}}\right)$; (c) evolução da taxa de resfriamento $(\dot{\mathrm{T}})$ e (d) progresso do gradiente térmico $(\mathrm{G})$ com a posição para a liga Bi-1,5\%Ag solidificada unidirecionalmente.

A Figura 4 mostra que uma estrutura colunar prevaleceu até uma posição de aproximadamente $50 \mathrm{~mm}$ a partir da interface metal/molde no fundido. Observa-se que para posições acima de $50 \mathrm{~mm}$, como não é objetivo do trabalho estudar a transição colunar equiaxial (TCE), as avaliações microestruturais estão restritas até $50 \mathrm{~mm}$.

Leis experimentais de crescimento do tipo $\lambda=a \dot{T}^{-0,55}$ e $\lambda=a \mathrm{~V}^{-2 / 3}$ foram determinadas para a liga $\mathrm{Bi}-1,5 \% \mathrm{Ag}$ conforme mostra a Figura 5 . Cada ponto no gráfico representa a média dos espaçamentos dendríticos $\left(\lambda_{1}, \lambda_{2}\right)$ e as barras de erro são os valores máximo e mínimo. A variação de $\lambda_{1} \mathrm{com}$ a taxa de resfriamento (Tं) foi caracterizada pelo expoente $-0,55$, como relatado por Rocha e coautores [15] para o crescimento celular de ligas $\mathrm{Sn}-\mathrm{Pb}$ solidificadas unidirecionalmente sob condições de fluxo de calor transitório, enquanto a variação de $\lambda_{2} \mathrm{com}$ a velocidade da isoterma liquidus $\left(V_{L}\right)$ foi caracterizada por um expoente de $-2 / 3$.

* Contribuição técnica ao $69^{\circ}$ Congresso Anual da ABM - Internacional e ao 14ํㅡㄹ ENEMET - Encontro Nacional de Estudantes de Engenharia Metalúrgica, de Materiais e de Minas, 21 a 25 de julho de 2014, São Paulo, SP, Brasil. 

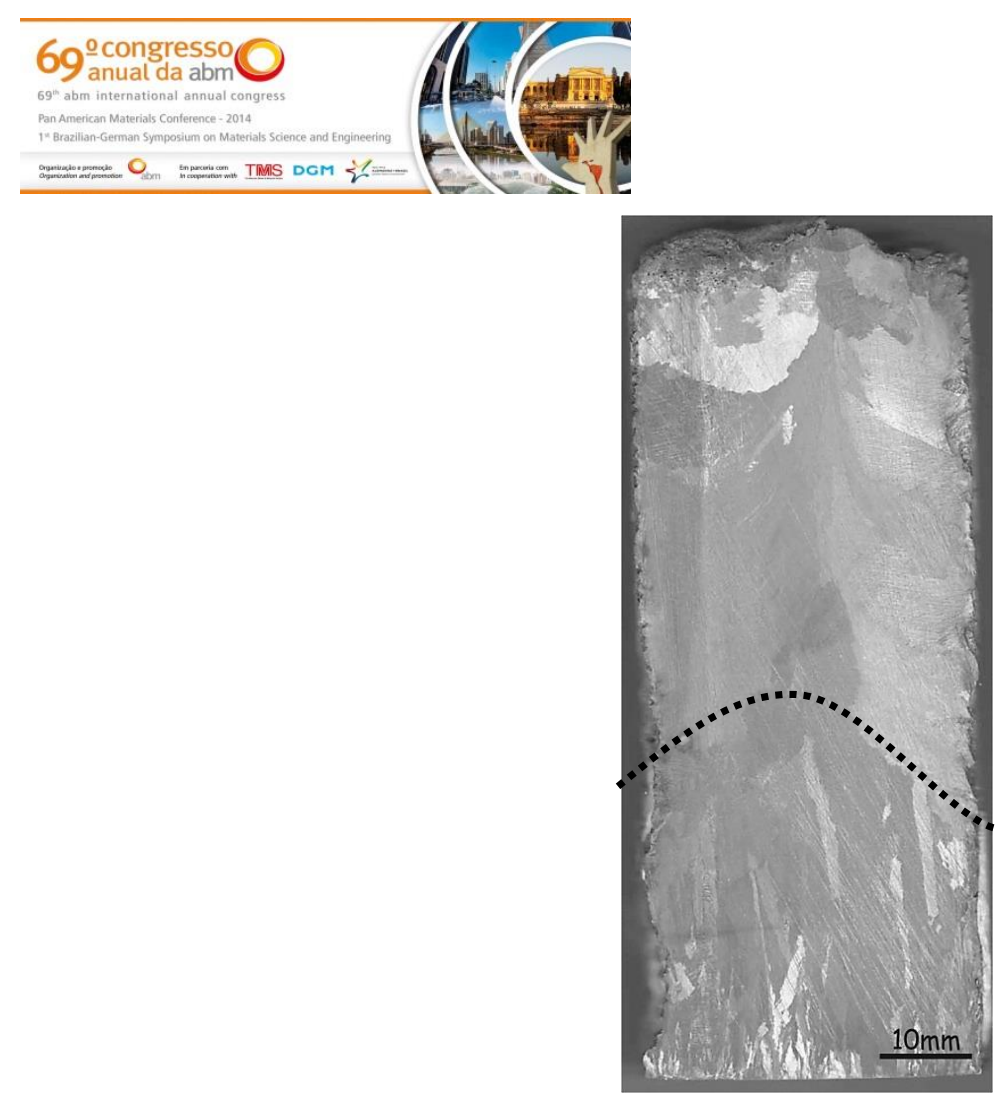

Figura 4. Macroestrutura da liga hipoeutética $\mathrm{Bi}-1,5 \% \mathrm{Ag}$ ressaltando a interface entre a região colunar (até $\pm 50 \mathrm{~mm}$ ) e a região equiaxial.

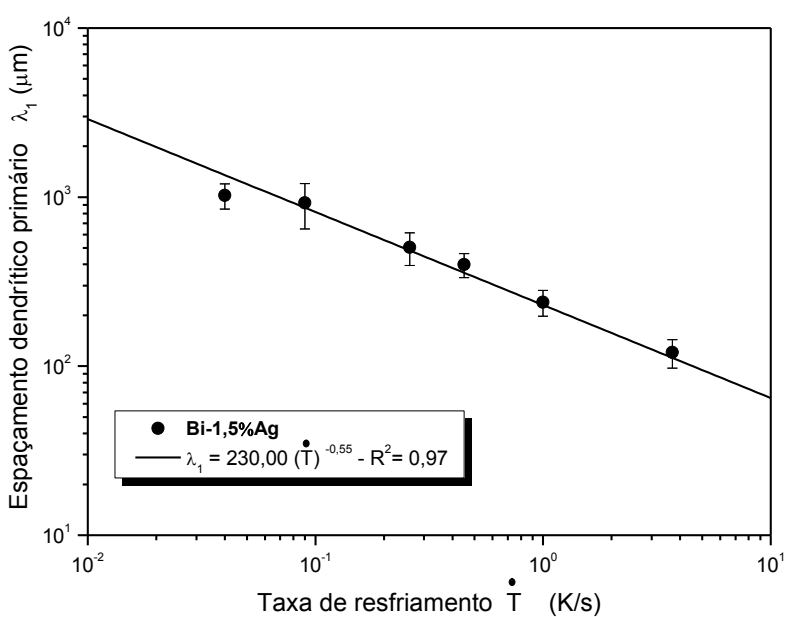

(a)

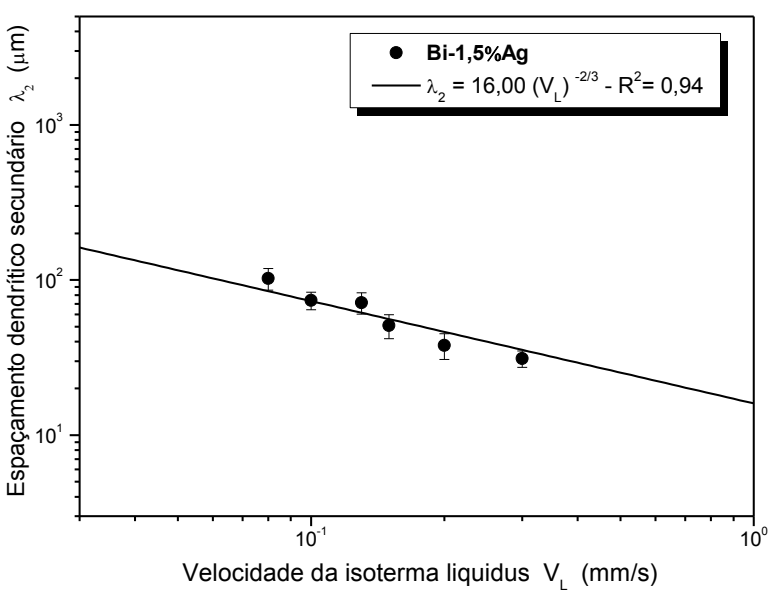

(b)

Figura 5. Variação dos espaçamentos dendríticos $\left(\lambda_{1}, \lambda_{2}\right)$ com (a) a taxa de resfriamento (广்) e (b) velocidade de isoterma liquidus $\left(\mathrm{V}_{\mathrm{L}}\right)$ para a liga $\mathrm{Bi} 1,5 \% \mathrm{Ag}$.

As microestruturas apresentadas na Figura 6 referem-se apenas a essa região de crescimento colunar mencionada anteriormente. Observando as microestruturas transversais, verifica-se uma microestrutura formada por dendritas facetadas ricas em $\mathrm{Bi}$ circundadas por uma mistura eutética $(\mathrm{Bi}+\mathrm{Ag})$ [16,17]. Analisando as microestruturas longitudinais, nota-se a prevalência de direcionalidade (na direção de extração de calor) das dendritas ricas em $\mathrm{Bi}$ (região mais clara). Além disso, fica claro o aumento gradativo da estrutura quando se observa os valores dos espaçamentos dendríticos médios primário $\left(\lambda_{1}\right)$ e secundário $\left(\lambda_{2}\right)$, passando de $\lambda_{1}=120,6 \mu \mathrm{m}$ e $\lambda_{2}=31,1 \mu \mathrm{m}$ para a posição $5 \mathrm{~mm}$, chegando a $\lambda_{1}=1023,8 \mu \mathrm{m}$ e $\lambda_{2}=102,2 \mu \mathrm{m}$ para a posição $50 \mathrm{~mm}$.

A Figura 7 mostra a variação do teor de $\mathrm{Ag}$ (\% em peso) ao longo do lingote $\mathrm{Bi}$ $1,5 \% \mathrm{Ag}$. Nota-se que para as posições mais distantes da base refrigerada o teor de

* Contribuição técnica ao $69^{\circ}$ Congresso Anual da ABM - Internacional e ao 14ํㅡㄹ ENEMET - Encontro Nacional de Estudantes de Engenharia Metalúrgica, de Materiais e de Minas, 21 a 25 de julho de 2014, São Paulo, SP, Brasil. 


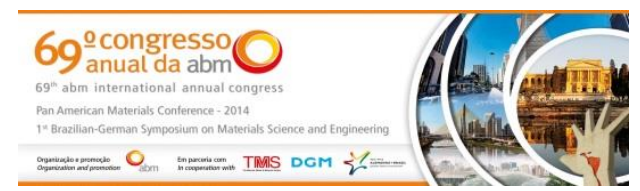

Ag é maior do que a composição nominal $(1,5 \% \mathrm{Ag})$, enquanto que para as posições iniciais do lingote (interface metal/molde) a quantidade de Ag é menor. Este perfil de macrossegregação traduz uma segregação do tipo normal, onde o coeficiente de redistribuição de soluto é menor que a unidade $(k<1)$.

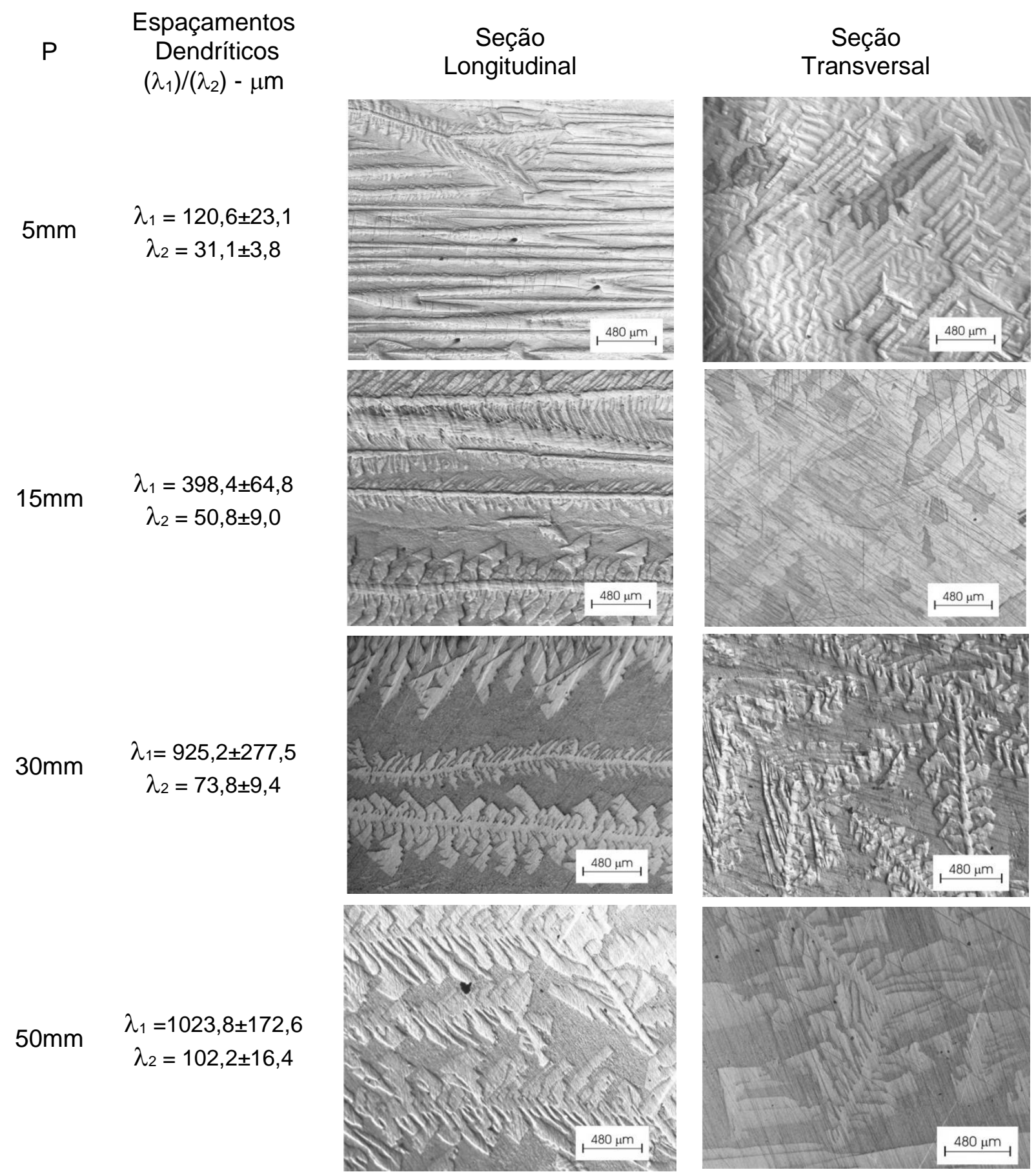

Figura 6. Microestruturas transversais e longitudinais típicas da liga hipoeutética $\mathrm{Bi}-1,5 \% \mathrm{Ag}$. $\mathrm{P}$ é a posição a partir da interface metal/molde.

* Contribuição técnica ao $69^{\circ}$ Congresso Anual da ABM - Internacional e ao 14ํㅡㄹ ENEMET - Encontro Nacional de Estudantes de Engenharia Metalúrgica, de Materiais e de Minas, 21 a 25 de julho de 2014, São Paulo, SP, Brasil. 

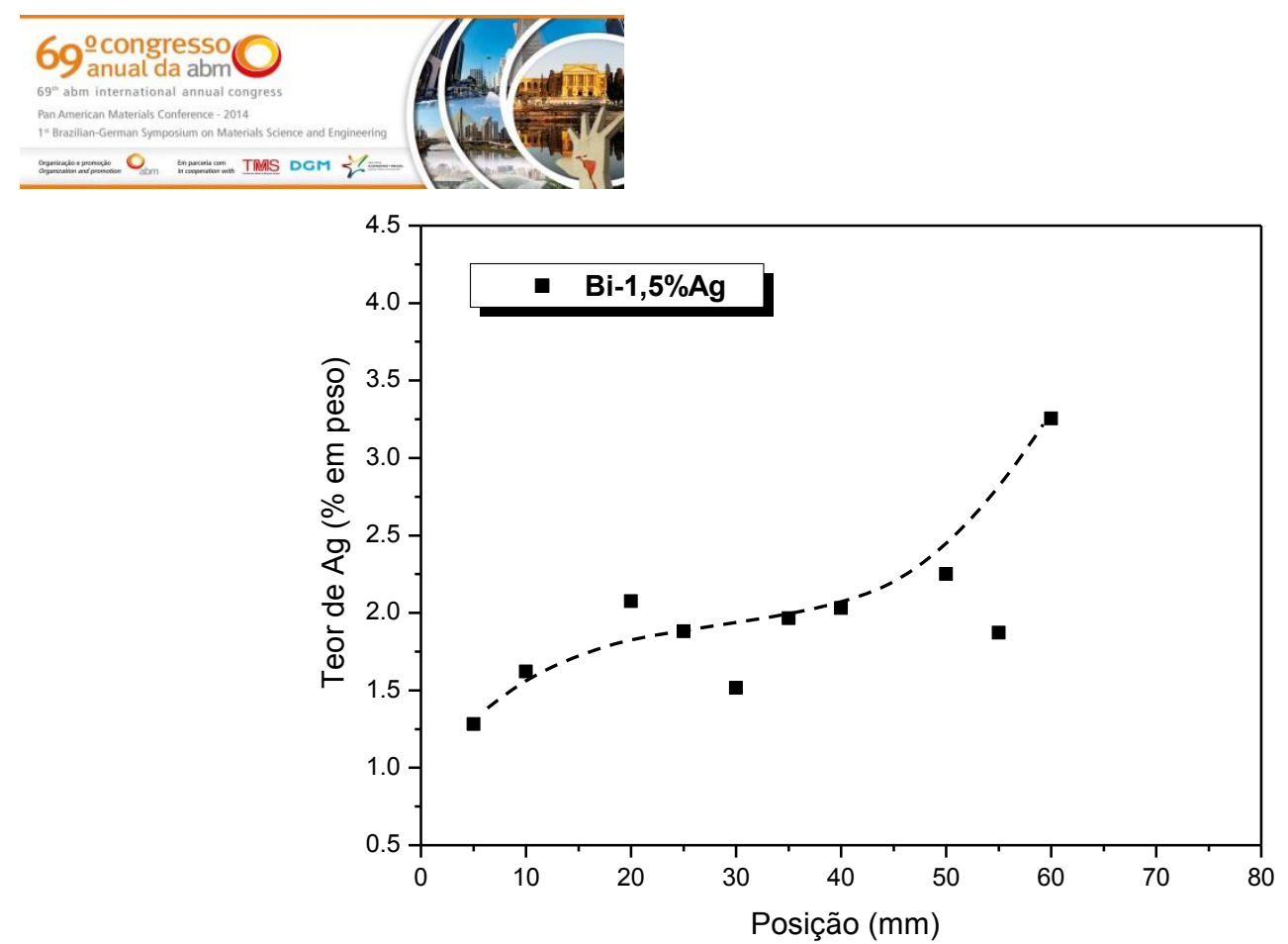

Figura 7. Perfil da macrossegregação de $\mathrm{Ag}$ na liga $\mathrm{Bi}-1,5 \% \mathrm{Ag}$ obtido por fluorescência de raios- $\mathrm{X}$.

A Figura 8 apresenta a correlação experimental obtida entre os valores de dureza Vickers (HV) e os correspondentes valores de espaçamento dendrítico secundário ג2. Os pontos representam os valores médios de dureza HV e as barras de erro os desvios padrão para cada região analisada. Uma equação do tipo Hall-Petch modificada foi determinada para a liga $\mathrm{Bi}-1,5 \% \mathrm{Ag}$. Observando a Figura 8 nota-se que quanto menor $\lambda_{2}$, maiores são os valores de dureza, chegando a um patamar de dureza entre 14-16 HV para a estrutura mais refinada da liga $\mathrm{Bi}-1,5 \% \mathrm{Ag}$, ou seja, valores de $\lambda 2^{-1 / 2}>0,16$. A associação de menores espaçamentos dendríticos primários e secundários com menores teores de prata $(\mathrm{Ag})$ parecem favorecer a existência de tal patamar. A Ag (na forma pura) possui dureza de $24,5 \mathrm{HB}$ ao passo que a dureza do bismuto puro é de 94,2 HB [18].

Os valores de dureza encontrados neste trabalho estão no mesmo nível ou até superiores àqueles encontrados na literatura no que diz respeito aos valores de HV relatados para a liga eutética $\mathrm{Sn}-37 \% \mathrm{~Pb}$ [19] e também para a liga $\mathrm{Pb}-5 \% \mathrm{Sn}$ (20). Siewert [19] mostrou que a convencional liga Sn-37\%Pb apresenta durezas médias entre 12 e $13 \mathrm{HV}$, enquanto Kamal et al [20] descreveram que a $\mathrm{Pb}-5 \% \mathrm{Sn}$ exibiu durezas em torno de $7 \mathrm{HV}$, indicando que a liga em análise pode ser uma alternativa para substituir as ligas $\mathrm{Sn}-\mathrm{Pb}$ em aplicações onde o requisito de dureza seja exigido.

* Contribuição técnica ao $69^{\circ}$ Congresso Anual da ABM - Internacional e ao 14ํㅡㄹ ENEMET - Encontro Nacional de Estudantes de Engenharia Metalúrgica, de Materiais e de Minas, 21 a 25 de julho de 2014, São Paulo, SP, Brasil. 

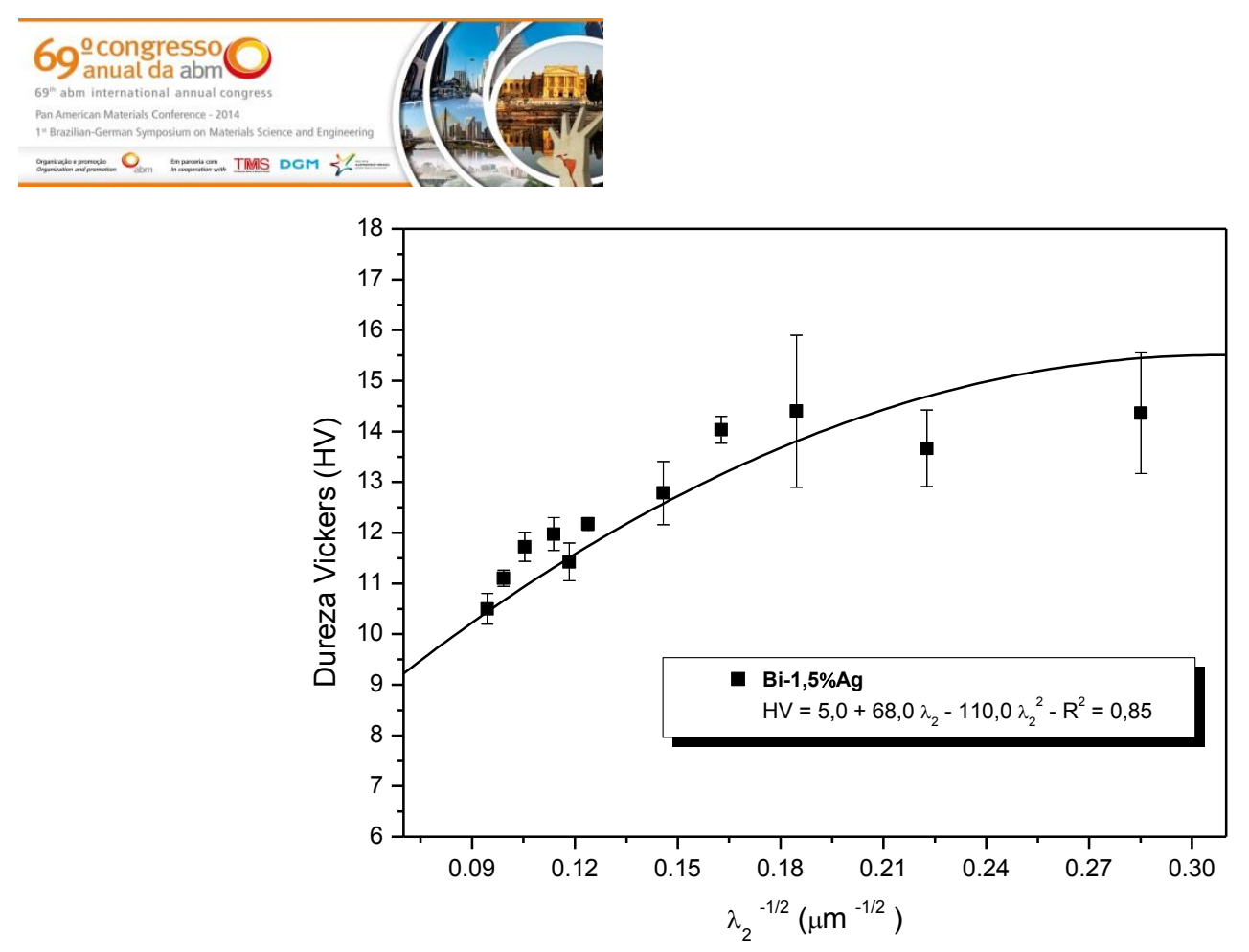

Figura 8. Dureza Vickers (HV) em função de $\lambda_{2}{ }^{-1 / 2}$ para a liga hipoeutética Bi-1,5\%Ag.

\section{CONCLUSÕES}

De acordo com os resultados obtidos para a liga hipoeutética $\mathrm{Bi}-1,5 \% \mathrm{Ag}$ solidificada unidirecionalmente em regime transitório de fluxo de calor, pode-se concluir:

- As evoluções da velocidade da isoterma liquidus $\left(\mathrm{V}_{\mathrm{L}}\right)$, da taxa de resfriamento $(\dot{T})$ e do gradiente térmico $(G)$ com a posição mostraram que os valores de $V L$, † e G diminuem à medida que distância da base refrigerada é aumentada. Os valores de $V_{L}$ ficaram entre $0,6 \mathrm{~mm} / \mathrm{s}$ e $0,08 \mathrm{~mm} / \mathrm{s}$, enquanto $\dot{T}$ apresentou valores entre $14,0 \mathrm{~K} / \mathrm{s}$ e $0,02 \mathrm{~K} / \mathrm{s}$;

- As análises microestruturais mostraram que um crescimento dendrítico prevaleceu ao longo da região de análise da liga $\mathrm{Bi}-1,5 \% \mathrm{Ag}$ a partir da interface metal/molde. A microestrutura típica consistiu de dendritas ricas em $\mathrm{Bi}$ circundadas por uma região eutética $(\mathrm{Bi}+\mathrm{Ag})$. Estudos posteriores são necessários para maior detalhamento do crescimento eutético;

- Foi observado que a lei experimental (entre medidas de dureza Vickers e valores de espaçamento dendrítico secundário) desenvolvida para representar o comportamento de dureza da liga $\mathrm{Bi}-1,5 \% \mathrm{Ag}$ é adequada, visto o alto valor do coeficiente de correlação, $R=0,85$. Verificou-se também que quanto menor o $\lambda_{2}$, maior o valor de dureza, mostrando um patamar de dureza para valores de $\lambda_{2}{ }^{-1 / 2}$ superiores a 0,16 . Quando comparam-se os valores de dureza da liga estudada com aqueles encontrados para as ligas $\mathrm{Sn}-37 \% \mathrm{~Pb}$ e $\mathrm{Pb}-5 \% \mathrm{Sn}$, nota-se que o nível de dureza do lingote $\mathrm{Bi}-1,5 \% \mathrm{Ag}$ é satisfatório e, portanto, pode ser uma alternativa para substituição de sistemas $\mathrm{Sn}-\mathrm{Pb}$ em aplicações onde a propriedade de dureza é necessária.

\section{Agradecimentos}

Os autores agradecem o apoio financeiro provido pela Fundação de Amparo à Pesquisa do Estado de São Paulo (FAPESP - 2013/08259-3 e 2013/13030-5) e pelo Conselho Nacional de Desenvolvimento Científico e Tecnológico (CNPq).

\footnotetext{
* Contribuição técnica ao $69^{\circ}$ Congresso Anual da ABM - Internacional e ao 14ํㅡㄹ ENEMET - Encontro Nacional de Estudantes de Engenharia Metalúrgica, de Materiais e de Minas, 21 a 25 de julho de 2014, São Paulo, SP, Brasil.
} 


\section{REFERÊNCIAS}

1 Abtew M; Selvaduray G. Lead-free Solders in Microelectronics. Materials Science and Engineering, 2000; 27(5-6): 95-141.

2 El-Daly AA, Hammad AE. Development of high strength Sn-0,7Cu solders with the addition of small amount of $\mathrm{Ag}$ and In. Journal of Alloys and Compounds, 2011; 509 (34): 8554-8560.

3 Li D; Liu C; Conway PP. Characteristics of intermetallics and micromechanical properties during thermal ageing of $\mathrm{Sn}-\mathrm{Ag}$-Cu flip-chip solder interconnects. Materials Science and Engineering A, 2005; 391(1-2): 95-103.

$4 \mathrm{Ma} \mathrm{H}$; Suhling JC. A review of mechanical properties of lead-free solders for electronic packaging. Journal Materials Science, 2009; 44(5): 1141-1158.

5 Zeng G, Mcdonald S, Nogita K. Development of high-temperature solders: Review. Microelectronics Reliability, 2012; 52: 1306-1322.

6 Watson J, Castro G. High-Temperature Electronics Pose Design and Reliability Challenges, Analog Dialogue, p. 46-04, 2012.

7 Bismarck LS; Cheung N; Garcia A; Spinelli JE. Thermal Parameters, Microstructure, and Mechanical Properties of Directionally Solidified Sn-0.7wt\%Cu Solder Alloys Containing 0 ppm to 1000 ppm Ni. Journal of Electronic Materials, 2013; 42: 179-191.

8 Pereira PD; Spinelli JE; Garcia A. Combined effects of Ag content and cooling rate on microstructure and mechanical behavior of $\mathrm{Sn}-\mathrm{Ag}$-Cu solders. Materials and Design, 2013; 45: 377-383.

9 Garcia A. Solidificação: Fundamentos e Aplicações. $2^{\mathrm{a}}$ ed. Campinas: Editora da Unicamp, 2007, 399 p.

10 Silva BL, Araújo IJC, Silva WS, Goulart P, Garcia A, Spinelli JE. Correlation between dendrite arm spacing and microhardness during unsteady-state directional solidification of Al-Ni alloys. Philosophical Magazine Letters, 2011, 91(5): 337-343.

11 Canté MV, Spinelli JE, Cheung N, Garcia A. The Correlation Between Dendritic Microstructure and Mechanical Properties of Directionally Solidified Hypoeutectic Al-Ni Alloys. Metallurgical Materials Internacional, 2010; 16: 39-49.

12 Rosa DM. Estruturas Celulares, Transição Celular/Dendrítica e Estruturas Dendríticas na Solidificação Unidirecional Transitória. 2007. 199 p. Tese (Doutorado em Engenharia Mecânica) - Faculdade de Engenharia Mecânica, Universidade Estadual de Campinas (UNICAMP), Campinas, 2007.

13 Gündüz M, Çadirli E. Directional solidification of aluminium-copper alloys. Materials Science and Engineering A, 2002; 327(2): 167-185.

14 Mccartney DG, Hunt JD. Measurements of cells and primary dendrite arm spacing in directionally solidified aluminium alloys. Acta Metallurgica, 1981; 29: 1851-1863.

15 Rocha OL, Siqueira CA, Garcia A. Heat flow parameters affecting dendrite spacings during unsteady state solidification of $\mathrm{Sn}-\mathrm{Pb}$ and $\mathrm{Al}-\mathrm{Cu}$ alloys. Metallurgical and Materials Transaction A, 2003; 34(4): 995-1006.

16 Song J, Chuang $\mathrm{H}, \mathrm{Wu} \mathrm{Z}$. Interfacial reactions between Bi-Ag high-temperature solders and metallic substrates. Journal of Electronic Materials, 2006; 55: 1041-1049.

17 J. Song; H. Chuang; T. Wen. Thermal and tensile properties of Bi-Ag alloys. Metallurgical and Materials Transactions A, 2007; 38A: 1371-1375.

18 Samsonov GV. Handbook of the physicochemical properties of the elements. New York: Springer, US; 1968.

19 Siewert Tet al 2002. Database for Solder Properties with Emphasis on New Lead-free Solder. Disponível em: http://www.metallurgy.nist.gov/solder/NIST_LeadfreeSolder_v4.pdf

20 Kamal M et al 2001. Effect of Ternary Addition on Characteristics of Pb-Sn Base Alloys. Egyptian Journal of Solids, 2001; 24(2):161-170.

\footnotetext{
* Contribuição técnica ao $69^{\circ}$ Congresso Anual da ABM - Internacional e ao 14ํㅡㄹ ENEMET - Encontro Nacional de Estudantes de Engenharia Metalúrgica, de Materiais e de Minas, 21 a 25 de julho de 2014, São Paulo, SP, Brasil.
} 